\title{
Pale green lethal disorder in apple (Malus) is caused by a mutation in the PHYLLO gene which is essential for phylloquinone (vitamin $\left.K_{1}\right)$ biosynthesis
}

\author{
Benjamin Orcheski ${ }^{1} \cdot{\text { Robert } \text { Parker }^{2} \cdot \text { Susan Brown }}^{1}$
}

Received: 20 August 2015 / Revised: 3 November 2015 / Accepted: 13 November 2015 /Published online: 20 November 2015

(C) The Author(s) 2015. This article is published with open access at Springerlink.com

\begin{abstract}
Pale green lethal (PGL) is a recessive genetic disorder of apple (Malus) characterized by severe chlorophyll deficiency and seedling lethality. Following germination, seedlings cannot photosynthesize and do not survive beyond the cotyledon stage. PGL carriers are prevalent in cultivated apple (Malus $\times$ domestica Borkh.) germplasm, and a cross between two heterozygotes results in $25 \%$ lethality. This high mortality rate represents a significant loss of time, resources, and effort. It also causes segregation distortion which complicates genetic analysis of linked traits. We mapped PGL to the top of Linkage Group 16. Using a combination of genetic and biochemical methods, we identified the apple ortholog of PHYLLO (MdPHYLLO) as the gene responsible for PGL disorder. PHYLLO encodes a multifunctional enzyme that catalyzes key steps in the biosynthesis of phylloquinone (vitamin $\mathrm{K}_{1}$ ). PGL seedlings do not produce phylloquinone yet can be
\end{abstract}

Communicated by D. Chagné

This article is part of the Topical Collection on Genome Biology

Electronic supplementary material The online version of this article (doi:10.1007/s11295-015-0956-9) contains supplementary material, which is available to authorized users.

Benjamin Orcheski

bbo5@cornell.edu

Robert Parker

rsp3@cornell.edu

Susan Brown

skb3@cornell.edu

1 School of Integrated Plant Science, Section of Horticulture, New York State Agricultural Experiment Station, Cornell University, Geneva, NY 14456, USA

2 Division of Nutritional Sciences, College of Human Ecology, Cornell University, Ithaca, NY 14853, USA kept alive and healthy through exogenous application of phylloquinone. The PGL allele of MdPHYLLO contains a mutation that alters a highly conserved amino acid in one of the protein's enzymatic modules. This mutation is present in all PGL carriers tested and absent from all non-carriers. Reasons why the PGL allele of MdPHYLLO is so widespread in cultivated apple are discussed.

Keywords Apple $\cdot$ Chlorophyll $\cdot$ Chloroplast $\cdot$ Malus . PHYLLO . Phylloquinone

\section{Introduction}

Cultivated apple (Malus $\times$ domestica Borkh.) is an economically important fruit crop grown throughout temperate regions. Apple germplasm is genetically and phenotypically diverse, owing in part to multiple Malus species contributing to the cultivated apple and obligate outcrossing due to selfincompatibility (Velasco et al. 2010; Wu et al. 2013; Cornille et al. 2014). Diversity within cultivated apple is a great benefit to breeders, and older cultivars are used extensively to develop new, superior apple varieties (Brown 2012). One of the challenges in apple breeding is a recessive disorder called pale green lethal (PGL), characterized by a severe chlorophyll deficiency in which plants are unable to photosynthesize (Alston 1976; Way et al. 1976). Seedlings with PGL are readily identified in progeny due to their light green cotyledons and leaves, of which only one or two develop (Fig. 1). Two weeks after germination, the true leaves turn necrotic followed by the cotyledons, and seedlings die within a month.

PGL is at a single locus, and plants homozygous $(l l)$ for the recessive allele "l" develop PGL symptoms (Klein et al. 1961; Way et al. 1976). When two PGL carriers are crossed, $25 \%$ of the progenies die soon after germination. The high mortality 


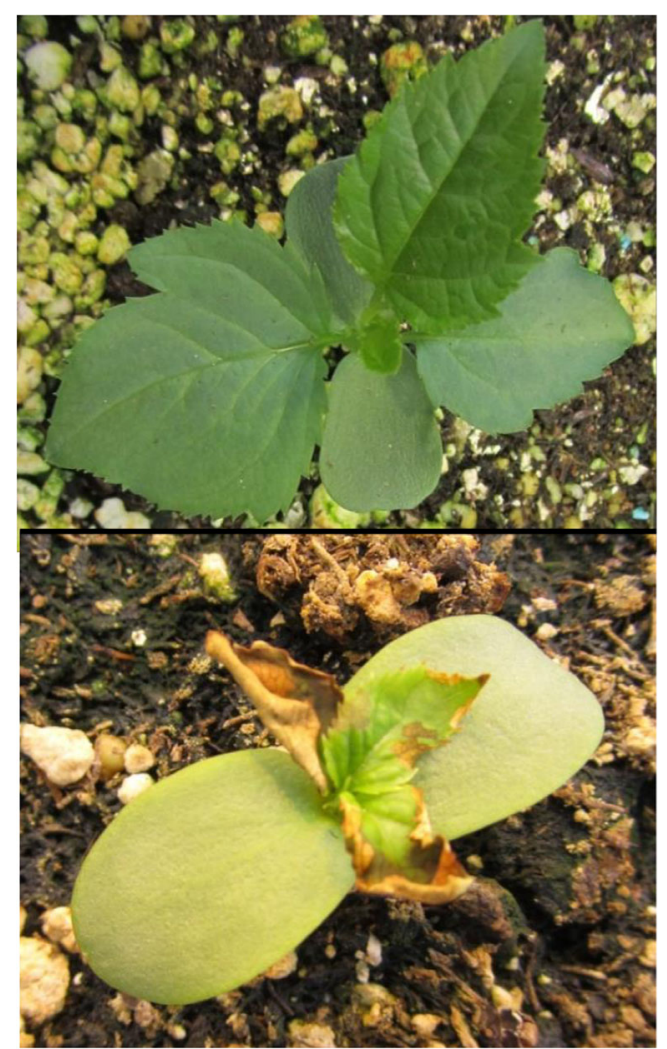

Fig. 1 Wild-type and pale green lethal seedlings. Three-week-old seedlings. Top -WT; bottom-PGL

rate of PGL represents a substantial loss of time and resources for breeders. PGL also creates segregation distortion which confounds genetic mapping and interpretation of the inheritance of linked economically important traits. PGL carriers are prevalent in apple germplasm and include many cultivars used frequently for breeding such as 'Golden Delicious,' 'Fuji,' and 'Jonathan.' This makes PGL more likely to arise in breeding populations.

PGL was first described by Crane and Lawrence (1930, 1933) who successfully selfed the apple variety 'Reverend W. Wilks.' In that progeny population, albino seedlings segregated in a roughly $3: 1$ green to albino ratio. Klein et al. (1961) expanded the known number of confirmed PGL carriers using large progeny populations, demonstrating that $\mathrm{PGL}$ was a recessive disorder at a single locus. Test crosses to known PGL carriers have expanded our knowledge of varieties containing the PGL allele (Brown 1958; Yoshida et al. 1964; Alston 1976; Way et al. 1976; Piestrzeniewicz et al. 2006). Only two studies attempted to characterize the PGL locus on a molecular level. Manganaris and Alston (1988) reported that isozymes of acid phosphatase (ACP-1) and endopeptidase (ENP-1) were linked to the PGL locus, but they were unable to assign the isozymes to a linkage group. Hatsuyama et al. (2006) reported that an allele of SSR marker $\mathrm{CH} 05 \mathrm{c} 06$ co-segregated with PGL. The $\mathrm{CH} 05 \mathrm{c} 06$ SSR was mapped to the top of apple Linkage Group (LG) 16 in several independent populations (Celton et al. 2009; FernándezFernández et al. 2008; Patocchi et al. 2009). Genes controlling three major economically important traits: fruit acidity (malic acid), phenolic content/diversity, and bitter pit resistance (a calcium-related postharvest disorder) also reside in this region of LG 16 (Bai et al. 2012; Khan et al. 2012; Buti et al. 2015).

Phylloquinone (PhQ), 2-methyl-3-phytyl-1,4naphthoquinone, also called vitamin $\mathrm{K}_{1}$, is a molecule essential for the proper functioning of animals and plants (van Oostende et al. 2011). Vitamin $K_{1}$ in animals is used to modify calcium-binding proteins that are required for bone metabolism and blood coagulation (Dolnikowski et al. 2002; Gröber et al. 2014). The vast majority of PhQ in plants is in photosynthetic tissues such that animals obtain their vitamin $\mathrm{K}_{1}$ primarily through consuming green plant parts (Card et al. 2014). The primary purpose of $\mathrm{PhQ}$ in cyanobacteria, green algae, and plants is to serve as a cofactor for the electron transport chain through photosystem I (PSI) (Schöttler et al. 2011). Arabidopsis mutants defective in PhQ biosynthesis have a severe reduction in PSI activity and chlorophyll content (Shimada et al. 2005; Gross et al. 2006; Kim et al. 2008). Mutant seedlings are pale green, are unable to survive photoautotrophically, and die soon after germination.

Although the biosynthesis of $\mathrm{PhQ}$ is divided between the chloroplast and the peroxisome, virtually, all $\mathrm{PhQ}$ is located in the chloroplast where the final three steps take place (Lohmann et al. 2006; van Oostende et al. 2011; Reumann 2013). Ten enzymatic steps are required to make PhQ from the initial chorismate precursor molecules (van Oostende et al. 2011; Fatihi et al. 2015). Four steps are catalyzed by a large, multifunctional, and multimodule enzyme called PHYLLO (Gross et al. 2006). In plants and green algae, the PHYLLO enzyme comprises four modules that correspond to the first four reactions in the PhQ pathway (van Oostende et al. 2011). However, PHYLLO only performs three steps in plants because the menF module is nonfunctional and the reaction menF normally catalyzes is performed by a separate enzyme, isochorismate synthase (Gross et al. 2006; van Oostende et al. 2011). The four modules of PHYLLO are derived from four separate eubacterial genes: $m e n F, m e n D$, menC, and menH, which are part of an operon for the synthesis of menaquinone, a related molecule (Fig. 2a). Through evolution, these four genes (modules) became fused into a single nuclear-encoded gene, and the order of modules has been maintained in green algae and plants. Mutations that disrupt the PHYLLO function cause a complete loss of $\mathrm{PhQ}$ production in Arabidopsis, indicating that this enzyme is essential for photosynthesis (Shimada et al. 2005; Gross et al. 2006).

We identified a mutation in the apple ortholog of PHYLLO (MdPHYLLO) that we propose is responsible for PGL disorder. This mutation causes an amino acid substitution that likely renders the active site of the menH module of MdPHYLLO 
Fig. 2 Overview of MdPHYLLO structure and processing. a Intron-exon diagram of the MdPHYLLO gene showing the location of the four men_ modules that comprise the PHYLLO gene. The MdPHYLLO gene has 28 exons (black boxes). A point mutation at base 4505 (relative to the start codon) in exon 25 has been identified as the cause of PGL. $\mathbf{b}$ Alternative splicing between exons $7-8$ and $8-9$ results in three transcript variants which generate truncated proteins. Intronic sequence present in the processed transcript is bold underlined

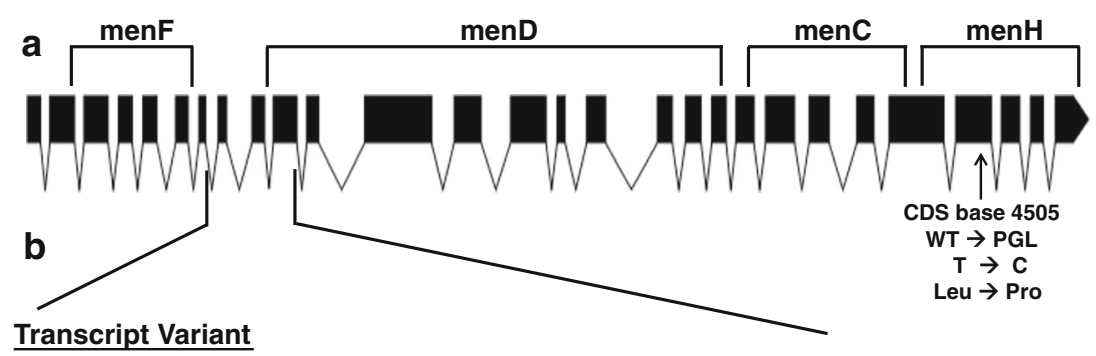

Full length (MdPHYLLO)

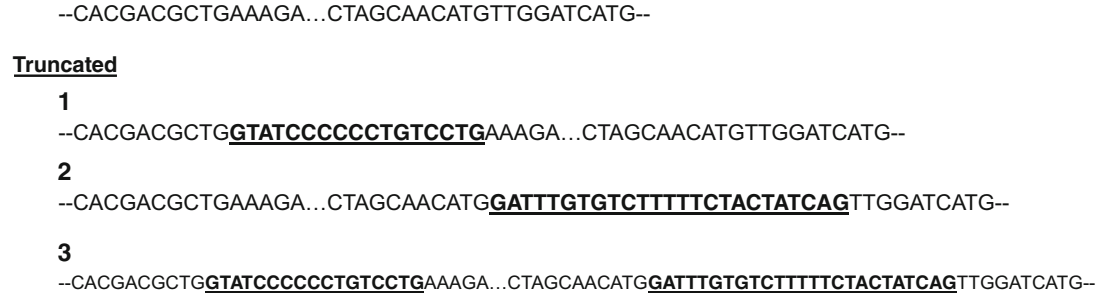

inactive. The mutant MdPHYLLO allele co-segregates with PGL in our mapping population and is present in all confirmed PGL carriers and absent from all non-carriers tested. PhQ accumulates in wild-type (WT) apple seedlings but cannot be detected in PGL seedlings. Application of a PhQ solution rescues the PGL phenotype and permits normal seedling growth. These data demonstrate the essential function of MdPHYLLO for apple seedling growth and development. We also discuss reasons why the PGL allele is prevalent in apple germplasm, its linkage to genes controlling important traits, and whether the mutation may impart some benefit to PGL carriers.

\section{Results}

\section{The PGL phenotype}

Upon germination, PGL seedlings are indistinguishable from WT but then fail to turn dark green (Fig. 1). There was a significant difference in fresh weight chlorophyll concentration between WT $\left(M=1.18 \mathrm{mg} \mathrm{g}^{-1}, \mathrm{SD}=0.17\right)$ and PGL $(M=$ $\left.0.71 \mathrm{mg} \mathrm{g}^{-1}, \mathrm{SD}=0.08\right)$ cotyledons; $t(8)=6.45, p=2 \mathrm{E}^{-4}$. This represents a $40 \%$ decrease in chlorophyll concentration in PGL seedlings compared with WT. By 2 weeks, WT seedlings have several fully developed true leaves while PGL seedlings have one to two stunted leaves that also lack chlorophyll. These true leaves then begin to turn necrotic, followed by the cotyledons, and seedlings die within a month.

\section{Mapping the PGL locus}

A PGL mapping population was generated by crossing two advanced breeding selections that were confirmed PGL carriers. A total of 157 progenies (92 WT and 65 PGL) were used for mapping, and the seedlings were scored as either PGL or WT after 3 weeks in the greenhouse.

Molecular markers for mapping were chosen based on their proximity to SSR CH05c06, which Hatsuyama et al. (2006) found to co-segregate with PGL. Four markers (Supplementary material; Table S1), all mapping to the top of LG 16, were obtained from the HIDRAS database (Gianfranceschi and Soglio 2004), as well as a cleaved amplified polymorphic sequence (CAPS) marker in the Mal gene, which is linked to CH05c06 (Bai et al. 2012). JoinMap v4.1 (Plant Research International, Wageningen, Netherlands) was used to resolve the progeny genotypic data into a single linkage group. Based on the genetic map, the PGL locus is located in a 1.7-cM interval between markers Mal and $\mathrm{CH} 05 \mathrm{c06}$ at the top of LG 16 (Fig. 3a). This interval is estimated to be $700 \mathrm{~kb}$ according to the 'Golden Delicious' apple genome assembly v1.0.

\section{Identifying PHYLLO as a candidate gene}

To identify PGL candidate genes, predicted transcripts located between Mal and $\mathrm{CH} 05 \mathrm{c06}$ were extracted from the Genome Database for Rosaceae (GDR). A total of 144 transcripts between the two markers were predicted from the apple genome v1.0. Transcript annotations were assessed for possible functions related to photosynthesis or plastids. Transcript function was also analyzed through queries to the NCBI Arabidopsis nucleotide collection using the tBLASTx algorithm.

The annotations and BLAST searches revealed three candidate genes with plastid- or photosynthesis-related functions: chloroplast unusual positioning 1 (CHUP1), heat shock protein 90.5 (HSP90.5), and PHYLLO. CHUP1, which controls the distribution of chloroplasts in the cell, has two paralogs in 
a

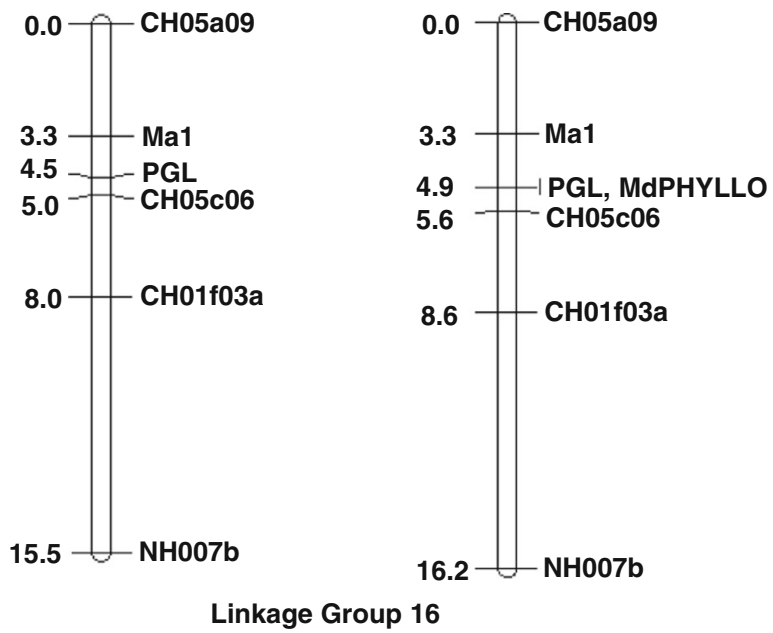

Fig. 3 Linkage maps of the pale green lethal locus. a The PGL locus is located at the top of Linkage Group 16 between markers Ma1 and CH05c06. b The MdPHYLLO gene co-segregates with the PGL phenotype

apple (LG 13; MDP0000314476 and LG 16; MDP0000320356), making it less likely to be the cause of a recessive disorder. HSP90.5 (MDP0000259301), which facilitates the entry of proteins into the plastid, is present in only one copy in apple. HSP90.5 complementary DNAs (cDNAs) were sequenced from PGL and WT haplotypes, translated, and aligned. No critical mutations were found after the translations, and any base substitutions were silent or resulted in a compatible amino acid. Thus, HSP90.5 was disregarded as a PGL candidate gene.

The MdPHYLLO (MDP0000194211) gene is present in a single copy, although the genome also contains a processed pseudogene (unanchored) and an unprocessed pseudogene (LG 13). The 'Golden Delicious' v1.0 genome assembly contains two alleles of the MdPHYLLO gene, which is not surprising since it is a PGL carrier. One allele contains a 3225-bp transposable element (TE) insertion at intron 20 that has $100 \%$ identity to apple 'RTE-1 Mad,' based on analysis with CENSOR transposon detection software (Genetic Information Research Institute, Mountain View, CA). RTE-1 Mad is a nonLTR retrotransposon prevalent in apple, with at least 900 copies in the 'Golden Delicious' genome. The full-length RTE-1 $M a d$ is $8.3 \mathrm{~kb}$, indicating that this insertion in the MdPHYLLO gene is only a fragment of the entire retrotransposon.

\section{Mapping MdPHYLLO}

Both parents of the PGL mapping population are heterozygous for the TE-containing MdPHYLLO allele; therefore, the presence/absence of the TE in MdPHYLLO was used to map the gene. The MdPHYLLO genotypic data was combined with the initial PGL genotyping data to generate a new linkage map (Fig. 3b). MdPHYLLO co-segregates with PGL such that all
PGL progenies are homozygous for the TE-containing allele. This led us to believe that the TE might be causing PGL by inducing some form of transcriptional gene silencing. To determine this, a number of confirmed PGL carrier and noncarrier varieties were tested for the presence of the TE. The TE-containing MdPHYLLO allele was amplified from both carriers and non-carriers, indicating that the TE insertion is not the cause of PGL.

\section{Structure and processing of MdPHYLLO}

To better characterize MdPHYLLO, the full-length gene and cDNA were cloned and sequenced from one mapping parent. The putative start and stop positions of MdPHYLLO were based on PHYLLO (PCP023290.1) in the pear (Pyrus communis L.) genome since this assembly is of higher quality than apple (Chagné et al. 2014). The MdPHYLLO coding sequence is 5079 bp and encodes a protein of 1692 amino acids. The MdPHYLLO gene spans 8947 and 12,338 bp in the WT and PGL alleles, respectively. Like Arabidopsis PHYLLO (AT1G68890), MdPHYLLO contains 28 exons, with the positions conserved between the two species.

The Arabidopsis PHYLLO gene is alternatively spliced to generate three transcripts, two of which generate truncated proteins due to premature stop codons (Gross et al. 2006). The alternative splicing occurs between exons 7 and 8, which corresponds to the region between the $\mathrm{menF}$ and menD modules of PHYLLO. We sequenced this region from 21 MdPHYLLO cDNA clones to determine if alternative splicing also occurs in apple. Nine of the clones produced the expected transcript, while 12 cDNAs showed alternative splicing. Alternative splicing occurred between exons 7 and 8 as well as exons 8 and 9 to generate three different transcript variants (Fig. 2b). The first transcript variant between exons 7 and 8 contains the intron sequence "GTATCCCCCCTGTCCTG" while the second variant between exons 8 and 9 contains the intron sequence "GATTTGTGTCTTTTTCTACTATCAG." Both of these intron sequences are present in the third variant. When translated, all three variants produce non-functional proteins because premature stop codons occur well before the menD module.

\section{Identification of a critical mutation in MdPHYLLO}

Twenty amino acid substitutions were present between the translated cDNAs of WT and PGL MdPHYLLO alleles. WT and PGL proteins were used as queries to the NCBI viridiplantae protein and nucleotide collections using the BLASTp and tBLASTn algorithms, respectively. For the majority of substitutions, the residues in the WT MdPHYLLO protein are not conserved across diverse plant families, indicating that they are likely not critical for the function of PHYLLO. However, in the WT protein, leucine at position 1502 (L1502) is highly conserved. Leucine was found at the 
equivalent position in 18 flowering plant families (basal angiosperms, monocots, and dicots), as well as lycophytes, bryophytes, chlorophytes, and over a dozen species of cyanobacteria (Fig. 4; Supplementary material; Table S2). One exception was in the Cucurbitaceae, where the equivalent position is a methionine. In the PGL MdPHYLLO protein, proline has been substituted for the leucine (L1502P).

The L1502P substitution occurs within the menH module of the MdPHYLLO gene. Therefore, we wanted to get a better understanding of the possible function of the leucine residue and how the substitution of proline might affect the enzyme. In bacteria and plants, the menH enzyme (module) catalyzes the third reaction in the biosynthesis of $\mathrm{PhQ}$ : conversion of 2-succinyl-5-enolpyruvyl-6-hydroxy-3-cyclohexene-1-carboxylic acid (SEPHCHC) to (1R,6R)-2-succinyl-6-hydroxy-2,4cyclohexadiene-1-carboxylic acid (SHCHC) (Jiang et al. 2008). High-resolution crystal structures of menH have been elucidated from Staphylococcus aureus and Escherichia coli (Dawson et al. 2011; Johnston et al. 2013). At the equivalent position of L1502, S. aureus has a leucine (L96) while E. coli has a methionine (M93). These residues are located in the middle of the third $\alpha$ helix and are seven residues upstream from the critical serine that forms the catalytic triad (Sun et al. 2014).

Proline is rarely found in the center of $\alpha$ helices because its cyclic structure prevents the hydrogen bonding necessary for helix formation (Woolfson and Williams 1990; Kim, and Kang 1999; Moore et al. 2003; Morgan and Rubenstein 2013). The lack of hydrogen bonding creates a kink which breaks the helical structure. We propose that the introduction of a proline into the middle of an $\alpha$ helix, so close to a critical active site residue, renders the menH module of the PGL MdPHYLLO protein non-functional. A non-functional menH module blocks downstream reactions in the $\mathrm{PhQ}$ pathway, preventing the biosynthesis of PhQ, making the L1502P MdPHYLLO allele a null.

\section{L1502P substitution in apple germplasm}

Based on our prediction that the L1502P substitution is the genetic basis of PGL, we wanted to determine the prevalence of the substitution within apple germplasm. We genotyped a number of varieties and selections that were previously confirmed as PGL carriers or non-carriers using test crosses or phenotyping of progeny (Brown, unpublished; Yoshida et al. 1964; Alston 1976; Way et al. 1976; Piestrzeniewicz et al. 2006). The L1502P substitution is caused by a transition at base 4505 (from start) from $\mathrm{T}$ to $\mathrm{C}$ in the MdPHYLLO coding sequence, which converts CTG (Leu) to CㅡG (Pro) (Supplementary material; Fig. S1). The SNP generates a restriction enzyme polymorphism whereby a BsrGI cut site (TGTACA) in the WT allele is lost in the PGL allele (CGGTACA). A CAPS marker, $\mathrm{CAPS}_{4505}$, was designed to genotype apple germplasm for the presence of the substitution.

A total of 70 accessions (37 PGL carriers and 33 noncarriers) were genotyped for the L1502P substitution. All 37 PGL carrier accessions were heterozygous for the "Leu" and "Pro" alleles of MdPHYLLO, and all 33 non-carriers were homozygous for the Leu allele (Supplementary material; Table S3). Although the restriction polymorphism does not distinguish which base $(\mathrm{A}, \mathrm{C}, \mathrm{G})$ is present in the PGL allele, all of them will abolish the $B s r G I$ restriction site and convert leucine to proline. The presence of a proline-coding MdPHYLLO allele in all PGL carriers tested, and its absence from any non-carriers, is further evidence that the L1502P substitution is the genetic basis of PGL.

\section{PhQ content in PGL and WT seedlings}

PhQ content in WT and PGL seedlings was determined using gas chromatography-mass spectroscopy (GC-MS) (Fig. 5). A substantial peak of PhQ was detected in the WT cotyledon samples. WT cotyledons had a PhQ concentration of $3.5 \mu \mathrm{g} /$ $\mathrm{g}$ fresh weight tissue (average of three biological replicates), a concentration in agreement with the range of PhQ content for other green leafy tissues (Booth et al. 1993; Damon et al. 2005). The amount of PhQ present in the PGL cotyledons was below the level of quantification, further supporting our view that MdPHYLLO is non-functional in PGL seedlings and that a lack of PhQ is the cause of PGL.

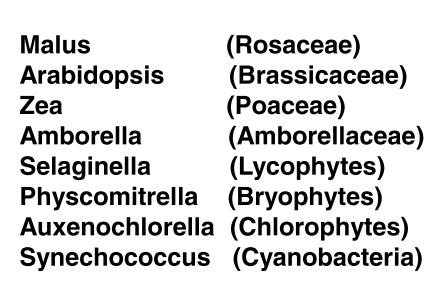

Fig. 4 The menH protein (module). The sequence conservation of the third alpha-helix (bracketed) of menH is evident in a diverse set of photosynthetic organisms. The leucine indicated by the arrow is

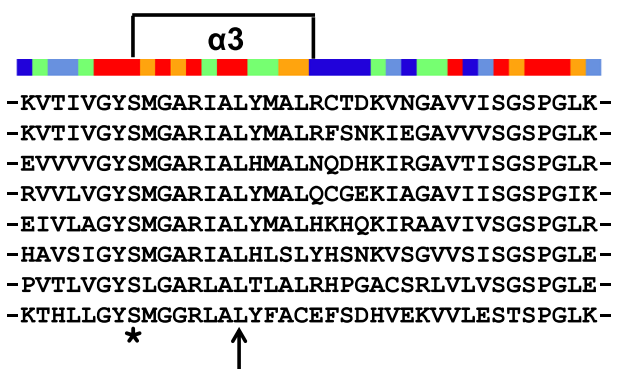

changed to a proline in the PGL allele of MdPHYLLO. The asterisk indicates the highly conserved serine that is part of catalytic triad of the menH protein 


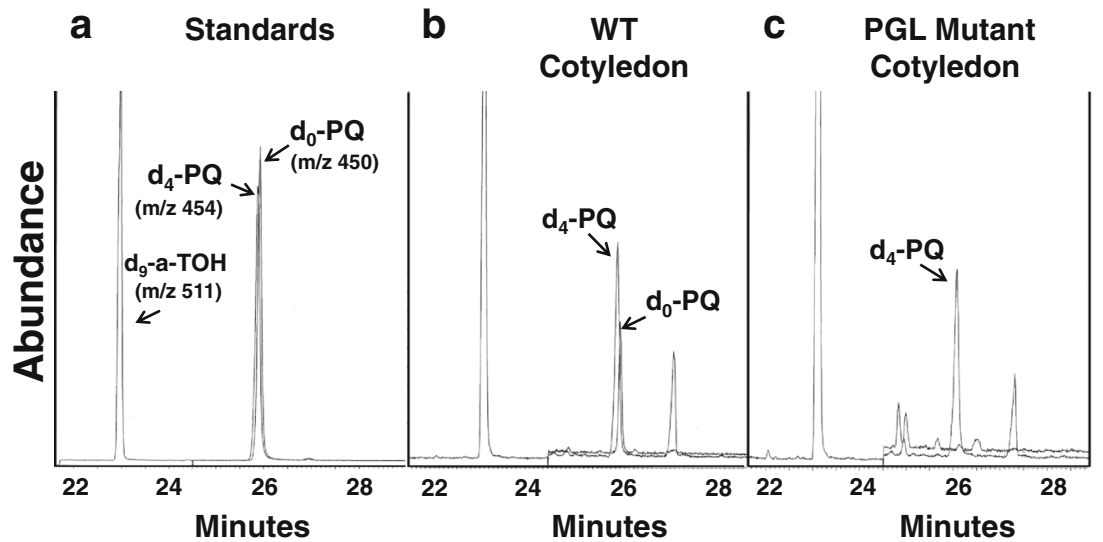

Fig. 5 Gas chromatography-mass spectroscopy chromatograms illustrating deficiency of phylloquinone in pale green lethal mutant cotyledons. a Mixture of standards: $d 9-a-T O H$ deuterium-labeled alpha- tocopherol (trimethylsilyl derivative, $m / z$ 511.4), $d 4-P Q$ deuteriumlabeled phylloquinone $(\mathrm{m} / \mathrm{z} 454.4), d 0-P Q$ unlabeled phylloquinone $(\mathrm{m} /$ $z$ 450.4). b Extract of WT cotyledons. $\mathbf{c}$ Extract of PGL mutant cotyledons

\section{PhQ rescue of PGL seedlings}

A $2 \%(v / v)$ PhQ solution was applied to PGL seedlings to determine if it could rescue the PGL phenotype. The solution was applied with a cotton swab to the leaves and cotyledons of PGL seedlings from three different cross combinations. A control set of non-treated PGL seedlings as well as treated and non-treated WT seedlings were also analyzed. The solution was applied every day for the first 3 weeks of growth, then every other day afterwards. The non-treated PGL seedlings died within a month, while the treated PGL seedlings turned green and developed true leaves. PhQ-treated PGL seedlings continued to recover, and by 2 months' time, they resembled WT seedlings (Fig. 6a). The only difference was that treated PGL seedlings were slightly smaller than the WT seedlings, and in a few seedlings, the apical meristem died back and the lateral meristems produced multiple branches. Treated and non-treated WT seedlings had normal growth indicating no detrimental effects of $\mathrm{PhQ}$ application. These results further confirm that a lack of PhQ is the cause of PGL.

Newly expanding leaves from 3-month-old PhQ-treated PGL and untreated WT seedlings were measured for chlorophyll content. There was a significant difference in fresh weight chlorophyll concentration between WT $(M=$ $\left.0.74 \mathrm{mg} \mathrm{g}^{-1}, \mathrm{SD}=0.18\right)$ and treated PGL $\left(M=1.34 \mathrm{mg} \mathrm{g}^{-1}\right.$, $\mathrm{SD}=0.46)$ seedlings; $t(28)=-5.81, p=3.02 \mathrm{E}^{-6}$. This represents an $80 \%$ increase in chlorophyll content following PhQ treatment. A $2 \%$ solution of PhQ did not register any absorbance, indicating that the higher values in treated PGL seedlings did not come from $\mathrm{PhQ}$ residue.

After 4 months in the growth chamber, the PhQ application was concluded and treated PGL seedlings were transferred to nursery beds. Seedlings continued to put out new growth, but the expanding leaves became chlorotic then necrotic and were often stunted (Fig. 6b). Leaves that had been supplied with PhQ remained green. After a month, chlorophyll content was measured from expanding leaves $\left(M=0.80 \mathrm{mg} \mathrm{g}^{-1}, \mathrm{SD}=0.22\right)$ as well as mature, chlorotic leaves $\left(M=0.37 \mathrm{mg} \mathrm{g}^{-1}, \mathrm{SD}=\right.$ 0.08). After PhQ application ended, chlorophyll content declined sharply in expanding leaves and was not significantly different from WT levels; $t(23)=-0.73, p=0.23$. Chlorophyll content of chlorotic, mature leaves was at levels significantly lower than even the PGL cotyledons; $t(16)=-7.09, p=$ $1.29 \mathrm{E}^{-6}$. Together, these data demonstrate that the increase in chlorophyll content in PhQ-treated PGL seedlings was temporary and dependent on continued PhQ application.

\section{PGL is linked to economically important traits}

One reason for the prevalence of deleterious alleles in cultivated crops is their linkage to economically important traits, a phenomenon called linkage drag (Labate and Robertson 2012). Our mapping of the PGL locus to the top of LG 16 allowed us to determine if any economically important traits are linked to the PGL locus. We found that Mal, a major determinant of fruit acidity in apple (Bai et al. 2012), is located only $1.6 \mathrm{cM}$ from the PGL locus (Fig. 3a). This area of LG 16 is also a QTL hotspot for phenolic content and diversity (Khan et al. 2012). Finally, a major QTL conferring bitter pit resistance is located in an interval surrounding SSR CH05c06 (Buti et al. 2015).

We investigated a possible relationship between the bitter pit resistance QTL and the PGL locus using progeny from a cross between 'Cameo' and 'Jonathan,' which are both PGL carriers. Buti et al. (2015) reported that 'Cameo' is not susceptible and demonstrated that the $\mathrm{CH} 05 \mathrm{c06} 101 \mathrm{bp}$ allele (104 bp in the GDR) segregates with bitter pit resistance. This is the same allele of $\mathrm{CH} 05 \mathrm{c} 06$ that segregates with PGL in our mapping population. We genotyped 14 PGL progenies from the 'Cameo' by 'Jonathan' cross and found that all the seedlings were homozygous for the $101 \mathrm{bp}$ allele and for the "Pro" PGL allele of MdPHYLLO. These data indicate that 


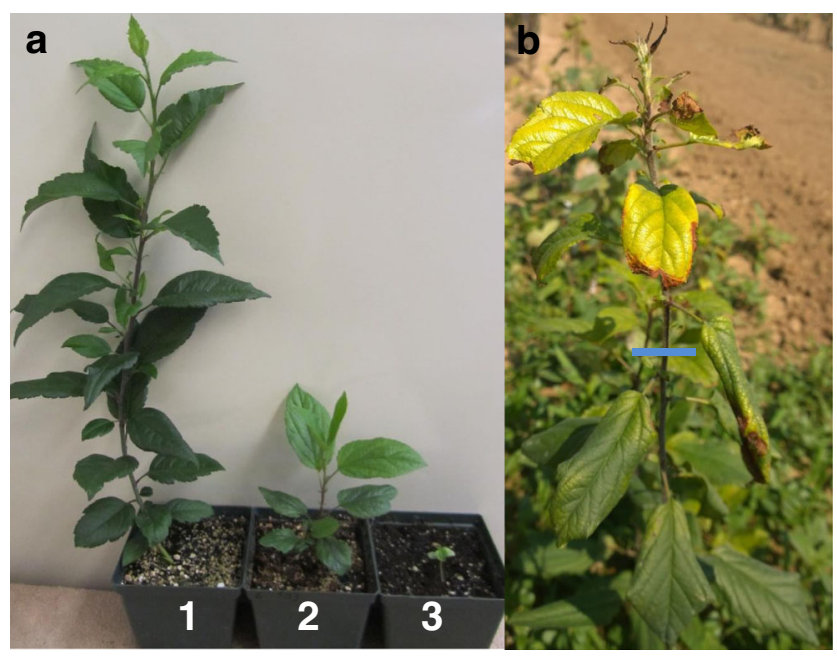

Fig. 6 Phylloquinone feeding experiment. a Comparison of seedlings from PhQ feeding experiment. $1-\mathrm{WT}$ seedling without PhQ application, 2-PGL seedling with PhQ application, 3-PGL seedling without PhQ application. b PGL seedling 1 month after feeding experiment stopped. The blue bar indicates the growth point at which the seedling was no longer supplied PhQ. Leaves that were supplied PhQ remain green weeks after treatment, while new growth becomes stunted and chlorotic and dies

the QTL for bitter pit resistance is in cis with the PGL allele of MdPHYLLO.

\section{Discussion}

\section{Absence of $\mathrm{PhQ}$ is responsible for PGL}

Pale green lethal (PGL) is an important recessive genetic disorder of apple (Malus). PGL seedlings are distinguished by their reduced chlorophyll content and inability to photosynthesize. Using a variety of genetic and biochemical methods, we determined that the cause of PGL is a deficiency of PhQ. $\mathrm{PhQ}$ is a plastid-localized molecule essential for photosynthesis in plants (Furt et al. 2010). PGL seedlings have PhQ content below the level of quantification by GC-MS. However, PGL seedlings supplemented with a PhQ solution recover from this disorder. The cotyledons and leaves of PhQ-treated seedlings become green, and the plants can photosynthesize and grow indefinitely, demonstrating the essential role of this molecule for plant growth.

A significant reduction in chlorophyll content is associated with PhQ mutants in Arabidopsis (Shimada et al. 2005; Gross et al. 2006; Kim et al. 2008), as well as the PGL phenotype of apple. The connection between chlorophyll and PhQ is not direct but appears to be based on the abundance of PSI. The Arabidopsis PhQ-deficient mutants, menA, menE, and PHYLLO, have reduced chlorophyll content and severely compromised PSI activity (Shimada et al. 2005; Gross et al. 2006; Kim et al. 2008). Alternatively, the Arabidopsis hcf 145 photosynthesis mutant also lacks chlorophyll and PSI activity but retains normal amounts of PhQ (Lezhneva and Meurer 2004). These data indicate that it is the deficiency of PSI activity and not PhQ that reduces chlorophyll content. Interestingly, application of PhQ to PGL seedlings increased the concentration of chlorophyll relative to untreated WT seedlings, suggesting that PhQ may stimulate production of chlorophyll. This might occur through reestablishment of PSI activity, or the shuttling of precursors or enzymes, such as geranylgeranyl diphosphate reductase, away from $\mathrm{PhQ}$ production and into the chlorophyll pathway (Schomburg and Schomburg 2013).

\section{A mutation in MdPHYLLO prevents PhQ biosynthesis}

Due to the severe loss of chlorophyll production and inability to photosynthesize, it is probable that the mutation causing PGL is in a gene required for photosynthetic or plastid functions. We have mapped PGL to a 1.7-cM interval at the top of LG 16. Within this interval, we identified the apple ortholog of PHYLLO which we have named MdPHYLLO. In higher plants, PHYLLO is essential for PhQ biosynthesis, as it catalyzes three steps in the pathway.

In our PGL mapping population, all PGL seedlings were homozygous for the TE-containing allele of MdPHYLLO, meaning that the gene co-segregates with the PGL phenotype. Analysis of WT and PGL MdPHYLLO cDNAs revealed a transition that causes an amino acid substitution within the menH module of the enzyme. The substitution, which occurs in an $\alpha$ helix near the active site, converts a leucine to a proline (L1502P). This leucine residue is conserved in a broad range of photosynthetic organisms from higher plants to cyanobacteria (Supplementary material; Table S2). We hypothesize that the substitution of proline prevents the formation of the $\alpha$ helix, thereby making the menH module of MdPHYLLO non-functional. The null MdPHYLLO blocks downstream steps to prevent the accumulation of PhQ in PGL seedlings.

We developed a CAPS marker to easily screen germplasm for the presence of the L1502P substitution. Seventy accessions that are confirmed as either PGL carriers or non-carriers were tested, and in all cases, the PGL carriers were heterozygous for the mutation (genotype Leu/Pro) while the noncarriers were homozygous (genotype Leu/Leu). These findings support our conclusion that the transition that converts leucine to proline is the mutation responsible for PGL disorder.

\section{PHYLLO structure and processing are conserved}

The structure and processing of the PHYLLO gene are conserved between Arabidopsis and Malus, with both genes containing 28 exons, and the exon-intron boundaries preserved. 
The genes encode proteins of similar size: 1716 amino acids in Arabidopsis and 1692 amino acids in apple. Interestingly, both PHYLLO genes are alternatively spliced between exons 7 and 8 , implying that this process serves an important function in these species and perhaps all higher plants. Gross et al. (2006) speculated that alternative splicing might regulate the amount of isochorismate precursor that flows into the PhQ and salicylic acid (SA) pathways. The alternative transcripts produce truncated, non-functional PHYLLO proteins, which would prevent the use of isochorismate for PhQ production, and shunt it into the SA pathway. Thereby, the plant could control the flow of isochorismate into the two pathways by controlling the ratio of functional and non-functional PHYLLO transcripts. However, this does seem a circuitous way to conserve isochorismate when the plant could simply control the transcription of PHYLLO.

\section{PGL in relation to apple breeding and research}

PGL is an impediment to apple breeding due to its high mortality, prevalence in the germplasm, and ability to confound mapping studies. By identifying the genetic basis of PGL, we provide apple breeders and researchers with a powerful tool. Using the $\mathrm{CAPS}_{4505}$ marker that we have developed, breeders can now screen their breeding material for the presence of PGL carriers. PGL carriers can be avoided if necessary, or additional progeny can be planted if it is known that PGL will be segregating in a cross.

The PGL mutation is interesting from an evolutionary standpoint and may lend insight into the history and spread of the cultivated apple (Malus $\times$ domestica Borkh.). As all of the PGL carriers tested contain the same mutation in the MdPHYLLO gene, it is likely that the mutant allele arose only once. This means that all of the PGL carriers are related through pedigree, and the mutation may be used to infer relationships when a pedigree is in doubt. Two accessions of Malus prunifolia (Willd.) Borkh., a species closely related to cultivated apple, were found to be PGL carriers (Supplementary material; Table S3). This means that either the PGL mutation arose before the divergence of these species or there has been interspecific gene flow. The PGL mutation is at least several hundred years old because 'Calville Blanc' which dates to the 1500 s is a carrier. The mutation has also spread extensively, as many European and North American varieties are PGL carriers.

Domesticated species often experience a genetic bottleneck when they are introduced to a new area (Bai and Lindhout 2007). Alston (1976) reported that the frequency of PGL carriers is much higher in North American-derived varieties than European varieties. One explanation is that a genetic bottleneck occurred during the introduction of cultivated apple to North America, whereby one or more of the founders was a PGL carrier.

\section{Prevalence of PGL in Malus germplasm}

PGL is a problem for apple breeders due to the prevalence of PGL carriers in the germplasm. Brown (1958) calculated that at least $35 \%$ of accessions in their collections were PGL carriers. Furthermore, the occurrence of PGL in breeding populations is exacerbated by extensive use of PGL carriers like 'Golden Delicious,' 'Jonathan,' and 'Fuji' as parents.

One possibility for the abundance of PGL carriers is that the PGL locus is linked to alleles of economically important genes. During the breeding process, these desirable alleles will be selected for, and if the PGL allele is tightly linked to them, it will be inadvertently selected, a process known as "linkage drag" (Labate and Robertson 2012). At least three economically important loci for fruit acidity, phenolic content/diversity, and bitter pit resistance are tightly linked to PGL (Bai et al. 2012; Khan et al. 2012; Buti et al. 2015).

Another explanation may be that in the heterozygote form, the PGL allele actually provides some benefit to the tree, a phenomenon called "heterozygote advantage" (Hedrick 2012). Isochorismate is the metabolic precursor molecule in the PhQ pathway, but SA, a plant hormone vitally important to plant defense, also requires isochorismate for its biosynthesis (Gross et al. 2006; Vlot et al. 2009). It is possible that in PGL carrier trees, the null allele of MdPHYLLO allows more isochorismate to flow into the SA pathway, thus heightening plant defense and providing an advantage for the tree. Krishna et al. (2012) reported that preharvest and postharvest applications of SA significantly reduce the incidence of many postharvest storage disorders. Wang and $\mathrm{Li}$ (2006) demonstrated that exogenous application of SA helped grape plants maintain calcium homeostasis under cold stress conditions. Bitter pit arises from long periods of cold storage and appears to be a calcium-related disorder (Volz et al. 2006). If PGL carriers produce more SA, they may be better suited to maintain calcium homeostasis and thus reduce the incidence of bitter pit. Given that they are both in cis, it is possible that the bitter pit resistance QTL reported by Buti et al. (2015) is due to the null $M d P H Y L L O$ allele and its effect on the SA pathway.

\section{Conclusion}

The seedling mortality and segregation distortion caused by pale green lethal (PGL) make it important in apple breeding. Our findings demonstrate that a mutation in MdPHYLLO, the apple ortholog of PHYLLO, is the genetic basis of PGL. The mutation alters a highly conserved amino acid which prevents the biosynthesis of $\mathrm{PhQ}$, a molecule essential for photosynthesis. PGL seedlings do not contain detectable levels of PhQ but can be kept alive through exogenous application of PhQ. We have developed a CAPS marker to easily genotype germplasm for the mutant allele which we mapped to the top of LG 
16. The mutant allele is present in all confirmed PGL carriers and absent from all non-carriers, indicating that it is a reliable method for apple breeders to identify PGL carriers in their breeding material. The marker is an important tool since PGL carriers are prevalent in apple germplasm.

\section{Experimental procedures}

\section{Plant growth and maintenance}

Apple (Malus $\times$ domestica Borkh.) seeds from the PGL mapping population and the 'Cameo' by 'Jonathan' population were stratified for 10 weeks at $4{ }^{\circ} \mathrm{C}$ before sowing. Seeds were germinated at room temperature, and in the spring, seedlings were grown in the greenhouse. Greenhouse conditions were as follows: 14-h light at $22{ }^{\circ} \mathrm{C}$ and 10 -h dark at $18{ }^{\circ} \mathrm{C}$, with ambient humidity. For the $\mathrm{PhQ}$ feeding experiment, seedlings were maintained in a growth chamber. Chamber conditions were as follows: ambient humidity, $650 \mu \mathrm{mol} \mathrm{m}{ }^{-2} \mathrm{~s}^{-1}$ light, $21{ }^{\circ} \mathrm{C}$, and a 16/8-h photoperiod. All seedlings were dosed with 2 tbsp of Osmocote fertilizer (Scotts, Marysville, $\mathrm{OH}$ ). Varieties and Malus species used for genotyping were grown at the USDA's National Plant Germplasm System, apple collection in Geneva, NY. Advanced selections were grown at the New York State Agricultural Experiment Station's research orchards in Geneva, NY.

\section{Sequence analysis}

Transcripts between markers $\mathrm{CH} 05 \mathrm{c} 06$ and $\mathrm{Mal}$ were downloaded from the GDR Whole Genome v1.0 Assembly (rosaceae.org/species/malus/malus_x_domestica/ genome_v1.0). The NCBI BLAST tool was used for homology searches. Sequence analysis and primer design were performed with the Lasergene Suite (DNAStar, Madison, WI). All primer sequences can be found in supplementary material; Table S1.

\section{Linkage analysis and map construction}

An $F_{1}$ mapping population of 157 individuals was made by crossing two advanced selections that are PGL carriers. Progeny were scored as WT or PGL in the greenhouse and genotyped with four SSR markers from the HIDRAS database as well as a CAPS marker (using HaeIII) for the Mal gene. An SV40 promoter primer sequence (https://mcb.berkeley.edu/ barker/dnaseq/free_stock primers) was added to the $5^{\prime}$ end of each SSR primer so they could be labeled during amplification with a FAM-labeled SV40 promoter primer. Labeled SSR PCR products were resolved on an ABI 3730xl DNA Analyzer (Applied Biosystems, Waltham, MA) and scored with Peak Scanner 2 software (Applied Biosystems, Waltham, MA). Genotypic data was converted into JoinMap "CP" (cross pollinator) codes according to parental genotypes and type of marker. A linkage map of the PGL locus was created with JoinMap 4.1 software using the recombination frequency grouping parameter and the maximum likelihood method.

\section{Chemical analysis of apple tissues}

Chlorophyll content was measured according to the protocol from Evans et al. (2012). Briefly, 50-100 mg of leaf tissue and $250 \mathrm{mg}$ of cotyledon tissue were immersed in 3 and $5 \mathrm{ml}$ of dimethylformamide (DMF), respectively. Samples were incubated overnight at $4{ }^{\circ} \mathrm{C}$ then centrifuged at $17,000 \mathrm{~g}$ for $5 \mathrm{~min}$. Absorbance was measured on a NanoDrop ND-1000 (Thermo Fisher, Waltham, MA) at 647 and $664 \mathrm{~nm}$. Fresh weight total chlorophyll $\left(\mathrm{Chl}_{\mathrm{a}}\right.$ and $\mathrm{Chl}_{\mathrm{b}}$ ) concentration was measured using the following equation: Chlorophyll $\left(\mathrm{mg} \mathrm{g}^{-1}\right.$ tissue $)=\left(\left(7.12 \mathrm{~A}_{664}+18.12 \mathrm{~A}_{647}\right) * \mathrm{~L}\right.$ DMF $) /(\mathrm{g}$ tissue $* 0.1-\mathrm{cm}$ path length). Two-sample Student's $t$ tests with unequal variances were performed on the chlorophyll concentration data. Significance was accepted at $p<0.05$, and values are noted in the text.

To measure PhQ content, cotyledons were excised, flash frozen in liquid nitrogen, and stored at $-80{ }^{\circ} \mathrm{C}$. Lipid extract from the cotyledons was silylated and analyzed by gas chromatography-mass spectroscopy as described by Bardowell et al. (2012) with some alterations. Gas chromatography temperature conditions were as follows: $150^{\circ} \mathrm{C}, 2$-min hold, $8{ }^{\circ} \mathrm{C}$ per minute to $250^{\circ} \mathrm{C}, 2$-min hold, $25^{\circ} \mathrm{C}$ per minute to $280^{\circ} \mathrm{C}$, 21-min hold. The internal standard was deuterated (d4) PhQ (Buchem BV, Apeldoorn, Netherlands), added as an ethanol solution prior to homogenization. Molecular ions monitored were $\mathrm{m} / \mathrm{z} 454.4$ (d4-PhQ) and $\mathrm{m} / \mathrm{z} 450.4$ (unlabeled PhQ).

\section{PhQ feeding}

A $2 \%(v / v)$ PhQ solution was applied to cotyledons and leaves with a cotton swab at the beginning of the dark cycle because PhQ is light sensitive. PhQ was applied every day for the first 3 weeks then every other day afterward. To make the solution, $1 \mathrm{ml}$ of PhQ (Alfa Aesar, Ward Hill, MA) dissolved in $2 \mathrm{ml}$

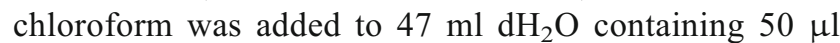
Tween-80. The mixture was shaken vigorously before application to keep PhQ in solution.

Acknowledgments The authors would like to thank K. Maloney for his technical assistance and care of the seedlings as well as E. Richards and M. Scanlon for reviewing the manuscript. This work was supported by the US Department of Agriculture, National Institute of Food and Agriculture Predoctoral Fellowship Grant-2015-67011-22787.

Data archiving statement The data reported here are archived and publicly available at the National Center for Biotechnology Information (NCBI; https://www.ncbi.nlm.nih.gov/). Accession numbers are as follows: WT PHYLLO cDNA-KT152850; PGL PHYLLO cDNA-KT152851; WT PHYLLO gene-KT152852; PGL PHYLLO 
gene-KT152853; WT HSP90.5 cDNA-KT152854; PGL HSP90.5 cDNA—KT152855; MdPHYLLO Processed Pseudogene-KT158468.

\section{Compliance with ethical standards}

Conflict of interest The authors declare that they have no conflict of interest.

Open Access This article is distributed under the terms of the Creative Commons Attribution 4.0 International License (http:// creativecommons.org/licenses/by/4.0/), which permits unrestricted use, distribution, and reproduction in any medium, provided you give appropriate credit to the original author(s) and the source, provide a link to the Creative Commons license, and indicate if changes were made.

\section{References}

Alston FH (1976) Dwarfing and lethal genes in apple progenies. Euphytica 25:505-514

Bai Y, Lindhout P (2007) Domestication and breeding of tomatoes: what have we gained and what can we gain in the future? Ann BotLondon 100:1085-1094

Bai Y, Dougherty L, Li M, Fazio G, Cheng L, Xu K (2012) A natural mutation-led truncation in one of the two aluminum-activated malate transporter-like genes at the Ma locus is associated with low fruit acidity in apple. Mol Genet Genomics 287:663-678

Bardowell SA, Duan F, Manor D, Swanson JE, Parker RS (2012) Disruption of mouse cytochrome p450 4f14 (Cyp4f14 gene) causes severe perturbations in vitamin E metabolism. J Biol Chem 287: 26077-26086

Booth SL, Sadowski JA, Weihrauch JL, Ferland G (1993) Vitamin $\mathrm{K}_{1}$ (phylloquinone) content of foods: a provisional table. J Food Compos Anal 6:109-120

Brown AG (1958) Pale green lethal in apple varieties. John Innes Institute 48th Annual Report 1957:6-7

Brown S (2012) Apples. In: Badnes ML, Byrne DH (eds) Handbook of plant breeding, 2012th edn. Springer, New York, pp 329-367

Buti M, Poles L, Caset D, Magnago P, Fernandez Fernandez F, Colgan RJ, Velasco R, Sargent DJ (2015) Identification and validation of a QTL influencing bitter pit symptoms in apple (Malus $\times$ domestica). Mol Breed. doi:10.1007/s11032-015-0258-9

Card DJ, Gorska R, Cutler J, Harrington DJ (2014) Vitamin K metabolism: current knowledge and future research. Mol Nutr Food Res 58: $1590-1600$

Celton JM, Tustin DS, Chagné D, Gardiner SE (2009) Construction of a dense genetic linkage map for apple rootstocks using SSRs developed from Malus ESTs and Pyrus genomic sequences. Tree Genet Genomes 5:93-107

Chagné D, Crowhurst RN, Pindo M, Thrimawithana A, Deng C, Ireland H, Fiers M, Dzierzon H, Cestaro A, Fontana P, Bianco L, Lu A, Storey R, Knäbel M, Saeed M, Montanari S, Kim YK, Nicolini D, Larger S, Stefani E, Allan AC, Bowen J, Harvey I, Johnston J, Malnoy M, Troggio M, Perchepied L, Sawyer G, Wiedow C, Won K, Viola R, Hellens RP, Brewer L, Bus VG, Schaffer RJ, Gardiner SE, Velasco R (2014) The draft genome sequence of European pear (Pyrus communis L. 'Bartlett'). PLoS One. doi:10.1371/journal. pone.0092644

Cornille A, Giraud T, Smulders MJ, Roldán-Ruiz I, Gladieux P (2014) The domestication and evolutionary ecology of apples. Trends Genet 30:57-65
Crane MB, Lawrence W (1930) Fertility and vigour of apples in relation to chromosome number. J Genet 22:153-163

Crane MB, Lawrence W (1933) Genetical studies in cultivated apples. J Genet 28:265-296

Damon M, Zhang N, Haytowitz D, Booth S (2005) Phylloquinone (vita$\min \mathrm{K}_{1}$ ) content of vegetables. J Food Compos Anal 18:751-758

Dawson A, Fyfe PK, Gillet F, Hunter WN (2011) Exploiting the highresolution crystal structure of Staphylococcus aureus $\mathrm{MenH}$ to gain insight into enzyme activity. BMC Structural Biol 11:19. doi:10. 1186/1472-6807-11-19

Dolnikowski GG, Sun Z, Grusak MA, Peterson JW, Booth SL (2002) HPLC and GC/MS determination of deuterated vitamin $\mathrm{K}$ (phylloquinone) in human serum after ingestion of deuteriumlabeled broccoli. J Nutr Biochem 13:168-174

Evans T, Song J, Jameson PE (2012) Micro-scale chlorophyll analysis and developmental expression of a cytokinin oxidase/ dehydrogenase gene during leaf development and senescence. Plant Growth Regul 66:95-99

Fatihi A, Latimer S, Schmollinger S, Block A, Dussault PH, Vermaas WF, Merchant SS, Basset GJ (2015) A dedicated type II NADPH dehydrogenase performs the penultimate step in the biosynthesis of vitamin K1 in Synechocystis and Arabidopsis. Plant Cell 27:17301741

Fernández-Fernández F, Evans KM, Clarke JB, Govan CL, James CM, Marič S, Tobutt KR (2008) Development of an STS map of an interspecific progeny of Malus. Tree Genet Genomes 4:469-479

Furt F, van Oostende C, Widhalm JR, Dale MA, Wertz J, Basset GJ (2010) A bimodular oxidoreductase mediates the specific reduction of phylloquinone (vitamin $\mathrm{K}_{1}$ ) in chloroplasts. Plant J 64:38-46

Gianfranceschi L, Soglio V (2004) The European project HiDRAS: innovative multidisciplinary approaches to breeding high quality disease resistant apples. Acta Hort 663:327-330

Gröber U, Reichrath J, Holick MF, Kisters K (2014) Vitamin K: an old vitamin in a new perspective. Derm Endo 6:1-6

Gross J, Cho WK, Lezhneva L, Falk J, Krupinska K, Shinozaki K, Seki M, Herrmann RG, Meurer J (2006) A plant locus essential for phylloquinone (vitamin K1) biosynthesis originated from a fusion of four eubacterial genes. J Biol Chem 281:17189-17196

Hatsuyama Y, Abe Y, Fukasawa-Akada T, Kon T, Igarashi M, Ueda T, Tsuyoshi Kudo T, Sato T, Masahiko Suzuki M (2006) Identification of DNA markers closely linked to pale green lethal in apple. Third Intl Rosaceae Genomics Conference

Hedrick PW (2012) What is the evidence for heterozygote advantage selection? Trends Ecol Evol 27:698-704

Jiang M, Chen X, Guo ZF, Cao Y, Chen M, Guo Z (2008) Identification and characterization of (1R,6R)-2-succinyl-6-hydroxy-2,4cyclohexadiene-1-carboxylate synthase in the menaquinone biosynthesis of Escherichia coli. Biochemistry-US 47:3426-3434

Johnston JM, Jiang M, Zhihong G, Baker EN (2013) Crystal structures of Escherichia coli native menH and two active site mutants. PLoS One. doi:10.1371/journal.pone.0061325

Khan SA, Chibon PY, de Vos RC, Schipper BA, Walraven E, Beekwilder J, van Dijk T, Finkers R, Visser RG, van de Weg EW, Bovy A, Cestaro A, Velasco R, Jacobsen E, Schouten HJ (2012) Genetic analysis of metabolites in apple fruits indicates an mQTL hotspot for phenolic compounds on Linkage Group 16. J Exp Bot 63:28952908

Kim MK, Kang YK (1999) Positional preference of proline in $\alpha$-helices. Protein Sci 8:1482-1499

Kim H, van Oostende C, Basset GJ, Browse J (2008) The AAE14 gene encodes the Arabidopsis o-succinylbenzoyl-CoA ligase that is essential for phylloquinone synthesis and photosystem-I function. Plant J 54:272-283

Klein LG, Way RD, Lamb RC (1961) The inheritance of a lethal factor in apples. P Am Soc Hort Sci 77:50-53 
Krishna H, Das B, Attri BL, Kumar A, Ahmed N (2012) Interaction between different pre- and postharvest treatments on shelf life extension of 'Oregon Spur' apple. Fruits 67:31-39

Labate JA, Robertson LD (2012) Evidence of cryptic introgression in tomato (Solanum lycopersicum L.) based on wild tomato species alleles. BMC Plant Biol. doi:10.1186/1471-2229-12-133

Lezhneva L, Meurer J (2004) The nuclear factor HCF145 affects chloroplast psaA-psaB-rps14 transcript abundance in Arabidopsis thaliana. Plant J 38:740-753

Lohmann A, Schöttler MA, Bréhélin C, Kessler F, Bock R, Cahoon EB, Dörmann P (2006) Deficiency in phylloquinone (vitamin $K_{1}$ ) methylation affects prenyl quinone distribution, photosystem I abundance, and anthocyanin accumulation in the Arabidopsis AtmenG mutant. J Biol Chem 281:40461-40472

Manganaris AG, Alston FH (1988) The acid phosphatase gene ACP-1 and its linkage with the endopeptidase gene ENP-1 and the pale green lethal gene $l$ in apple. Acta Hort 224:177-184

Moore DJ, Zhang L, Dawson TM, Dawson VL (2003) A missense mutation (L166P) in DJ-1, linked to familial Parkinson's disease, confers reduced protein stability and impairs homo-oligomerization. J Neurochem 87:1558-1567

Morgan AA, Rubenstein E (2013) Proline: the distribution, frequency, positioning, and common functional roles of proline and polyproline sequences in the human proteome. PLoS One. doi:10.1371/journal. pone. 0053785

Patocchi A, Fernandez-Fernanadez F, Evans K, Gobbin D, Rezzonico F, Boudichevskaia A, Dunemann F, Stankiewicz-Kosyl M, MathisJeanneteau F, Durel CE, Gianfranceschi L, Costa F, Toller C, Cova V, Mott D, Komjane M, Barbaro E, Kodde L, Rikkerink E, Gessler C, Van de Weg WE (2009) Development and test of 21 multiplex PCRs composed of SSRs spanning most of the apple genome. Tree Genet Genomes 5:211-223

Piestrzeniewicz C, Andziak J, Andziak E (2006) Emergence and mortality of seedlings in some apple (Malus $\times$ domestica Borkh.) progenies. J Fruit Orn Plant Res 14:81-89

Reumann S (2013) Biosynthesis of vitamin $\mathrm{K}_{1}$ (phylloquinone) by plant peroxisomes and its integration into signaling molecule synthesis pathways. In: del Rio LA (ed) Peroxisomes and their key role in cellular signaling and metabolism, 1st edn. Springer, Netherlands, pp 213-229

Schomburg D, Schomburg I (2013) Geranylgeranyl diphosphate reductase 1.3.1.83. In: Schomburg D, Schomburg I (eds) Springer handbook of enzymes, 2nd edn. Springer-Verlag, Berlin, pp 247-252

Schöttler MA, Albus CA, Bock R (2011) Photosystem I: its biogenesis and function in higher plants. J Plant Physiol 168:1452-1461

Shimada H, Ohno R, Shibata M, Ikegami I, Onai K, Ohto MA, Takamiya $\mathrm{K}$ (2005) Inactivation and deficiency of core proteins of photosystems I and II caused by genetical phylloquinone and plastoquinone deficiency but retained lamellar structure in a T-DNA mutant of Arabidopsis. Plant J 41:627-637

Sun Y, Yin S, Feng Y, Li J, Zhou J, Liu C, Zhu G, Guo Z (2014) Molecular basis of the general base catalysis of an $\alpha / \beta$-hydrolase catalytic triad. J Biol Chem 289:15867-15879

Van Oostende C, Widhalm JR, Furt F, Ducluzeau AL, Basset GJ (2011) Vitamin K1 (phylloquinone): function, enzymes, and genes. In: Rebeille F, Douce R (eds) Advances in botanical research, biosynthesis of vitamins in plants part B: vitamins B6, B8, B9, C, E, K, 1st edn. Elsevier, London, pp 230-255

Velasco R, Zharkikh A, Affourtit J, Dhingra A, Cestaro A, Kalyanaraman A, Fontana P, Bhatnagar SK, Troggio M, Pruss D, Salvi S, Pindo M, Baldi P, Castelletti S, Cavaiuolo M, Coppola G, Costa F, Cova V, Dal Ri A, Goremykin V, Komjanc M, Longhi S, Magnago P, Malacarne G, Malnoy M, Micheletti D, Moretto M, Perazzolli M, Si-Ammour A, Vezzulli S, Zini E, Eldredge G, Fitzgerald LM, Gutin N, Lanchbury J, Macalma T, Mitchell JT, Reid J, Wardell B, Kodira C, Chen Z, Desany B, Niazi F, Palmer M, Koepke T, Jiwan D, Schaeffer S, Krishnan V, Wu C, Chu VT, King ST, Vick J, Tao Q, Mraz A, Stormo A, Stormo K, Bogden R, Ederle D, Stella A, Vecchietti A, Kater MM, Masiero S, Lasserre P, Lespinasse Y, Allan AC, Bus V, Chagné D, Crowhurst RN, Gleave AP, Lavezzo E, Fawcett JA, Proost S, Rouzé P, Sterck L, Toppo S, Lazzari B, Hellens RP, Durel CE, Gutin A, Bumgarner RE, Gardiner SE, Skolnick M, Egholm M, Van de Peer Y, Salamini F, Viola R (2010) The genome of the domesticated apple (Malus $\times$ domestica Borkh.). Nature Genet 42:833-839

Vlot AC, Dempsey DA, Klessig DF (2009) Salicylic acid, a multifaceted hormone to combat disease. Annu Rev Phytopathol 47:177-206

Volz RK, Alspach PA, Fletcher DJ, Ferguson IB (2006) Genetic variation in bitter pit and fruit calcium concentrations within a diverse apple germplasm collection. Euphytica 149:1-10

Wang LJ, Li SH (2006) Salicylic acid-induced heat or cold tolerance in relation to $\mathrm{Ca}^{2+}$ homeostasis and antioxidant systems in young grape plants. Plant Sci 170:685-694

Way RD, Lamb RC, Pratt C, Cummins JN (1976) Pale green lethal gene in apple clones. J Am Soc Hort Sci 101:679-684

Woolfson DN, Williams DH (1990) The influence of proline residues on $\alpha$-helical structure. FEBS Lett 277:185-188

Wu J, Gu C, Khan M, Wu J, Gao Y, Wang C, Korban S, Zhang S (2013) Molecular determinants and mechanisms of gametophytic selfincompatibility in fruit trees of Rosaceae. Cr Rev Plant Sci 32:53-68

Yoshida Y, Tsuchiya S, Haniuda T (1964) Frequency of the albino seedling in some cross combinations of apple. Jap J Hort Sci 33:93-95 